$\Rightarrow$ THEORETICAL PHYSICS

\title{
Sound carries mass
}

High school physics classes teach that sound waves don't transport mass but instead perturb the medium they pass through by creating more-dense and less-dense regions. Now, Angelo Esposito and colleagues have shown that for superfluids, and more generally even for normal fluids and solids, this fundamental statement is not accurate. Sound waves do carry mass.

Using a theoretical approach called effective field theory, which is commonly used in particle and solidstate physics, the team calculated the mass carried by a sound wave packet propagating though a superfluid. The calculations show that sound waves carry a tiny negative mass, which means that in the presence of a gravitational field, such as that of the Earth, their trajectory is bent upwards. Esposito and colleagues found that sound waves also generate a small gravitational field.

Although the mass of sound waves is tiny, it could be measured

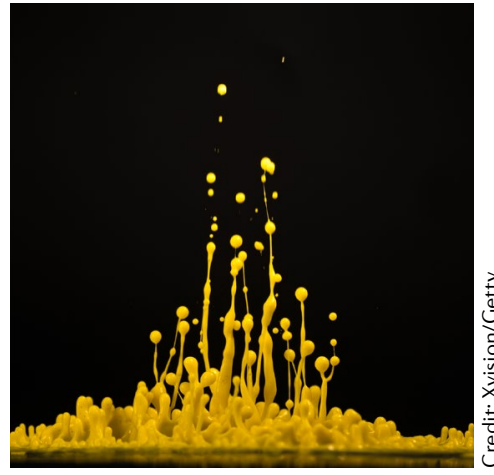

in experiments with cold molecular or atomic gases. The work might be relevant for neutron star dynamics, because gravitational fields would affect the physical properties of the superfluid stellar core.

\section{Anastasiia Novikova}

ORIGINAL ARTICLE Esposito, A., Krichevsky, R. \& Nicolis, A. Gravitational mass carried by sound waves. Phys. Rev. Lett. (in the press) FURTHER READING Esposito, A., Krichevsky, R. \& Nicolis, A. The mass of sound. Preprint at arXiv https://arxiv.org/abs/1807.08771 (2018) 